\title{
“Semillas Ambientales" en el Instituto Pedagógico Arturo Ramírez Montúfar (IPARM) de la Universidad Nacional de Colombia - Sede Bogotá.
}

Por: Torres Guerra Nubia ${ }^{\text {; }}$ Sánchez Medina Natalia ${ }^{2}$

\begin{tabular}{|l|}
\hline Recibido: $19-12-2008$ \\
\hline Aceptado: 01-06-2009 \\
\hline
\end{tabular}

Con la comunidad del IPARM se busca educar ambientalmente a partir de una labor pedagógica que logre satisfacer necesidades e intereses individuales y colectivos orientados hacia nuestro proyecto de vida comprometidos con la sociedad; al ser arte y parte con el entorno, cuidándolo a partir de su interactuar dentro del campus universitario. Lo anterior conlleva de alguna manera, a un cambio de valores y comportamientos que permite respetar la dinámica de los lugares en los cuales se desenvuelve su cotidianidad.

Se analiza y asume la concepción de Ambiente "Sistema complejo que es el resultado de las interacciones entre los factores naturales, sociales, tecnológicos y culturales de una sociedad, que incluye lo natural y lo construido por la humanidad" planteada en la Política Nacional de Educación Ambiental (PNEA, 2008).

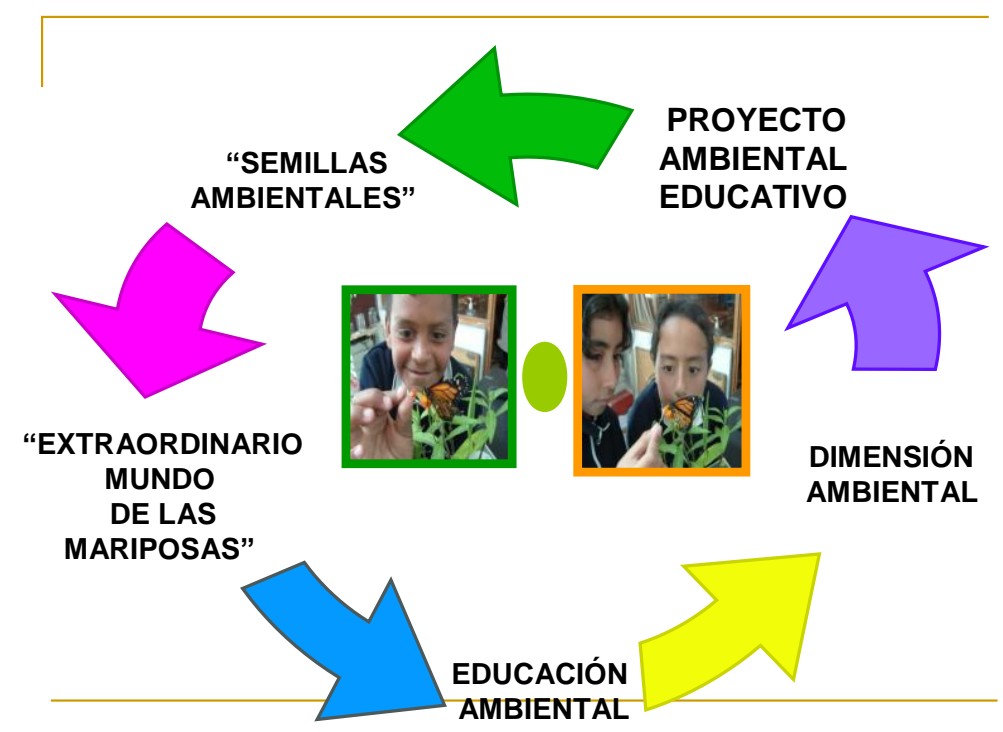

1 Lic. en química, Especialista en computación para la docencia.. M.Sc. Docencia de la Química, Docente Instituto Pedagógico Arturo Ramírez Montúfar, Universidad Nacional de Colombia, Sede Bogotá. Correo Electrónico: ntorresg@unal.edu.co.

2 Estudiante Licenciatura en Biología, Universidad Pedagógica Nacional. Docente Instituto Pedagógico Arturo Ramírez Montúfar, Universidad Nacional de Colombia, Sede Bogotá. Correo Electrónico: nasanchez@unal.edu.co 
La historia a nivel ambiental del IPARM se ha desarrollado internamente por iniciativa de los docentes, por temática temporal como contenidos de clase o producto de necesidades ambientales, este último aspecto ha contribuido en el establecimiento y elaboración de un diagnóstico del entorno próximo de la comunidad educativa. Además, se han divulgado las aproximaciones y conclusiones en la Pág. Web del IPARM y UN, se han establecido vínculos con la Asociación Colombiana para el avance de la Ciencia (ACAC), Fundación Alma, con el Sistema de Gestión Ambiental (SGA), el Programa de Ambiente y Desarrollo Sostenible del Departamento de Trabajo Social (PADS-TS), el Colectivo ambiental-Facultad de Agronomía de la Universidad Nacional de Colombia, con el Departamento de Biología de la Universidad Pedagógica Nacional en la Línea de Investigación Faunística y Conservación de Artrópodos cuyos integrantes abordan, entre otras actividades prácticas y de campo, el estudio de la Mariposa Monarca, se participa con actividades practicas con el Museo de la ciencia y el juego (UN) en la primera feria del conocimiento y la creatividad de la localidad 13 donde se da a conocer el Proyecto "Semillas Ambientales", entre otros.

El proyecto se planea y contextualiza de acuerdo a los objetivos, misión, visión y la concepción que maneja la Institución en torno a la formación en Ciencias Naturales y Educación Ambiental, este surge como una alternativa que busca fundamentalmente la sensibilización y adopción de un compromiso por parte de los estudiantes en relación a la protección y mejoramiento de su ambiente próximo y que incluye el aprender a cuidarse, cuidar y respetar al entorno por cada uno de sus miembros.

Se pretende desarrollar en los estudiantes autonomía, creatividad, habilidades y competencias básicas que les permita involucrarse con sus saberes por medio de una sensibilización a través de actividades de indagación, observación, identificación, relación y experimentación de tal forma que se vinculen principalmente con su responsabilidad con al ambiente y la construcción de una acción frente a las problemáticas actuales de tal forma que contrastan lo aprendido con su quehacer diario. Se llevan los significados a situaciones cotidianas y desde esa perspectiva, se establece la interdisciplinariedad con otras áreas del conocimiento, lo anterior implica un trabajo de permanente retroalimentación y se logra por medio de acciones que permiten evidenciar las relaciones ser humano-sociedad-naturaleza.

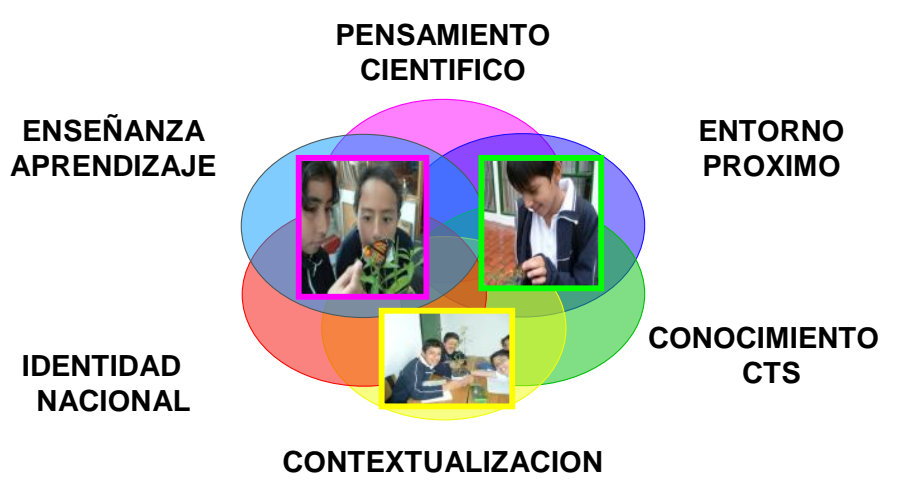


Lo expuesto incluye los aspectos generales temáticos de la propuesta pedagógica en Educación Ambiental "Semillas Ambientales" planeada, desarrollada y aplicada en subproyectos tales como la mariposa monarca (Danaus plexippus) de la Línea de Investigación Faunística y Conservación de Artrópodos de la Universidad Pedagógica Nacional, este espacio académico desde el año 2003 adelanta investigaciones de campo y laboratorio que implica la utilización de mariposas y otros insectos como instrumentos educativos para la enseñanza básica, secundaria y universitaria.

Las bases teóricas de la línea de Investigación se implementan en el IPARM desde el año 2007 con los estudiantes de Educación Básica y Media mediante el fortalecimiento del conocimiento biológico del organismo referenciado, se conciencia sobre el valor intrínseco y extrínseco del mismo, se reconoce a través de la adopción, observación y descripción de su ciclo de vida para evaluar en el proceso las actitudes afectivas, comportamentales y cognitivas que manifiesta el joven durante la convivencia con el organismo.

Se diseña un folleto que moviliza actitudes de tipo cognitivo, comportamental y afectivo con contenidos que dan cuenta de esquemas, dibujos y fotografías de algunos estudiantes de los grados, producto de la conceptualización e interpretación durante su cuidado; los estudiantes se identifican con el trabajo colectivo, asumen la construcción del conocimiento biológico de una especie como proceso fundamental, sostienen y observan la especie al vislumbrar algunas características de la crisálida o una Mariposa hembra sobre las flores de Asclepia curassavica, lo anterior se convierte en un paso para la conservación de un organismo específico, en este caso la mariposa monarca (Danaus plexippus).

En términos cognitivos, la mayoría de los estudiantes presentaron claridad en relación a los conceptos biológicos básicos de las mariposas para contrastar lo teórico y lo practico. Entre las explicaciones se resalta la siguiente: "La Asclepia curassavica es la planta de la cual se alimenta la mariposa monarca (Danaus plexippus).....", en su mayoría, emplearon nombres comunes y científicos para identificar las especies (tanto animal, en este caso la mariposa monarca como vegetal, la planta nutricia u hospedera de este organismo). Fueron cuidadosos e identificaron la forma de escritura de las especies según la jerarquía taxonómica. Este tipo de actitud cognitiva es importante, pues resulta indispensable conocer una especie, incluyendo las particularidades que la caracterizan y que la diferencian de otras al momento de asumir una postura de conservación y protección de estas especies.

En la Institución se ha fomentado el uso de la plataforma virtual (Blackboard), herramienta mediante la cual los estudiantes tienen la oportunidad de desarrollar actividades interactivas lúdico-recreativas en su aprendizaje en las clases. Este escenario de aprendizaje ha facilitado la consulta de material de distinta índole: esquemas y gráficos explicativos, diapositivas, y hojas de trabajo, videos, presentaciones de Power-Point para ser desarrolladas en las clases y fuera de estas. Teniendo en cuenta lo mencionado se diseñó una página WEB denominada "El extraordinario mundo de las mariposas", esta incluye los aspectos básicos en términos biológicos de la especie, acerca al estudiante, lo motiva al estudio de este organismo, tiene la 
posibilidad de formular preguntas que conllevan a que se remita a otras fuentes de información para resolverlas fortaleciendo el trabajo en equipo y favoreciendo la observación de las diferentes etapas (huevo, larva, pupa e Imago).

Dentro de los resultados obtenidos se puede afirmar que los estudiantes se familiarizan, dentro de la gran diversidad de insectos que habita nuestro planeta, con un grupo reducido entre los cuales se incluyen abejas, mariquitas y mariposas, al momento de graficar un insecto no lo hacen de manera independiente sino que vinculan o relacionan el entorno (hábitat) de este, sus hábitos alimenticios, relación con otros organismos al interior de un ecosistema, ciclo de vida, entre otros.

Durante la adopción del organismo se relacionan conceptos de la metamorfosis y esto contribuyó en las pautas de comportamientos, tales como el tipo de planta que se le debía suministrar, la cantidad del mismo, el tiempo que tardaba el ciclo de vida.

Esta fascinante experiencia ofrece innumerables ventajas como herramienta en la enseñanza de la biología, tales como; el poco tiempo que dura su ciclo de vida, los cambios radicales que son posibles observar durante cada etapa de su desarrollo, fáciles de mantener, manipular y obtener, entre otras.

\section{Bibliografía}

- ARANGO, N. 2002. Guía metodológica para la enseñanza de ecología en el patio de la escuela. Nueva York NY: Audubon, Programa para América Latina y el Caribe.

- Alcaldía mayor de Bogotá. 2004 - 2008. Política pública distrital de educación ambiental "Un Aprendizaje Conjunto para Una Ciudad Diversa". Plan de Desarrollo Distrital “Bogotá Sin Indiferencia: Un Compromiso Social Contra La Pobreza y La Exclusión".

- BENAYAS DEL ÁLAMO, J. 1992. Paisaje y Educación Ambiental: Evaluación de cambio de actitudes hacia el entorno. Ministerio de Obras Públicas y Transporte.

- GARCÍA, V. 2008. El aprendizaje significativo de David Paul Ausubel. Caja de herramientas para la administración de servicios de información.

- GIL, L. 2003. Fortalecimiento de actitudes y valores para el cuidado del entorno natural cercano a niños y niñas. Tesis de Grado. Universidad Pedagógica Nacional Facultad de Educación Departamento de Psicopedagogía Programa de preescolar Bogotá.

- LACUELA A. La enseñanza por proyectos: ¿mito o reto? Revista Iberoamericana de Educación. Número 16 - Educación Ambiental y formación: Proyectos y Experiencias.

- Ministerio del medio ambiente. 2002. Política nacional de educación ambiental (SINA). Ministerio de educación nacional.

- Ministerio de educación nacional. 1998. Ciencias Naturales y Educación Ambiental. Lineamientos Curriculares. Áreas Obligatorias y Fundamentales. Cooperativa Editorial Magisterio. MOLLÁ, MARÍ. “Desarrollo de actitudes en los alumnos". Psicología y pedagogía aplicadas. Vol. 16 1985, núm. 31: p. 31-44. 
- Ministerio de Educación Nacional 2008. Política Nacional de Educación Ambiental. CONAM. Consejo Nacional del Ambiente. Bogotá.

- NOVAK, J. D. Y GOWIN, D. B. 1988. Aprendiendo a aprender. Barcelona, España: Martínez Roca.

- PÉREZ R. 2001. Universidad Nacional Autónoma de México: Facultad de Filosofía y Letras. Programa de posgrado en Pedagogía. Seminario teoría y desarrollo curricular, evaluación y comunicación, nuevas tecnologías en el campo de la educación.

- RICO, M. 1992. El Aprendizaje de Valores en Educación Ambiental. Madrid. Ministerio de obras publicas y transporte.

- Secretaria de Educación. Propuesta para la implementación de la educación Ambiental en la Escuela en el Distrito Capital. Alcaldía Mayor de Santa fe de Bogota.

- Universidad Nacional de Colombia. Sede Bogotá. 2000. Proyecto Manejo Integral de Residuos Sólidos. 